\title{
Mepolizumab add-on therapy in a real world cohort of patients with severe eosinophilic asthma: response rate, effectiveness, and safety
}

Jermo Johannes van Toor, Sophie C. van der Mark, Jasper H. Kappen, J. C. C. M. In 't Veen \& Gert Jan Braunstahl

To cite this article: Jermo Johannes van Toor, Sophie C. van der Mark, Jasper H. Kappen, J. C. C. M. In 't Veen \& Gert Jan Braunstahl (2020): Mepolizumab add-on therapy in a real world cohort of patients with severe eosinophilic asthma: response rate, effectiveness, and safety, Journal of Asthma, DOI: 10.1080/02770903.2020.1723623

To link to this article: https://doi.org/10.1080/02770903.2020.1723623

Accepted author version posted online: 30 Jan 2020.

Published online: 12 Feb 2020.

Submit your article to this journal

山 Article views: 231

Q View related articles 5

View Crossmark data 


\title{
Mepolizumab add-on therapy in a real world cohort of patients with severe eosinophilic asthma: response rate, effectiveness, and safety
}

\author{
Jermo Johannes van Toor, $M D^{a, b} \mathbb{D}$, Sophie C. van der Mark, $\mathrm{MD}^{\mathrm{b}}$, Jasper H. Kappen, MSc, MD, PhD ${ }^{\mathrm{a}}$, \\ J. C. C. M. In 't Veen, $M D, P h D^{a}$, and Gert Jan Braunstahl, MD, PhD ${ }^{a, b}$

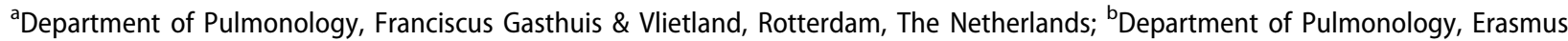 \\ MC, Rotterdam, The Netherlands
}

\begin{abstract}
Introduction: Severe eosinophilic asthma is an incapacitating disease. Mepolizumab, a humanized anti-interleukin-5 monoclonal antibody, proved to be effective as an add-on therapy in patients with severe eosinophilic asthma. However, only data from randomized controlled trials are available and real world data are lacking.

Methods: A retrospective observational longitudinal study was conducted in a real world cohort of patients with severe eosinophilic asthma treated with mepolizumab. The primary objective was to determine response rate, based on a global evaluation of treatment effectiveness by the treating pulmonologist. Secondary objectives were to assess exacerbation frequency, systemic maintenance glucocorticoid usage, Asthma Control Questionnaire (ACQ), lung function, and adverse events.

Results: Seventy-eight patients were included. Treatment with mepolizumab was considered beneficial and was therefore continued in $75.6 \%$ of patients 12 months from the initiation of mepolizumab. The most common reason for drop-out was insufficient response. Secondary objectives: 12 months from the initiation of mepolizumab there was a decrease of 3.2 (Cl 2.5-4.1; $p<0.001$ ) severe asthma exacerbations per year, a decrease of ACQ of 0.80 points (Cl $0.49-1.12 ; p<0.001)$, and an increase of $3.7(\mathrm{Cl} 0.3-7.2 ; p=0.034$ ) percent of predicted FEV1 compared to baseline. At baseline $51.3 \%$ of patients were treated with systemic glucocorticoid maintenance therapy, compared to $15.4 \%(p<0.001)$ of patients 12 months from the initiation of mepolizumab. No serious adverse events considered to be related to mepolizumab were reported.

Conclusion: This study confirms that mepolizumab add-on therapy is effective and safe in a real world cohort of patients with severe eosinophilic asthma.
\end{abstract}

\section{ARTICLE HISTORY}

Received 2 November 2019

Revised 17 January 2020

Accepted 26 January 2020

\section{KEYWORDS}

Asthma; eosinophils; interleukin-5; monoclonal antibodies; mepolizumab; real world

\section{Introduction}

Asthma is a heterogeneous inflammatory airway disease with symptoms varying over time and in intensity (1). In most cases, asthma can be well treated with a combination of inhaled glucocorticoids and a bronchodilator (1). However, 17 and $3.7 \%$ of patients with asthma suffer from difficult to treat asthma or severe asthma, respectively (2). Severe asthma is a subset of difficult-to-treat asthma, defined as asthma which requires treatment with high dose inhaled corticosteroids plus a second controller [and/or maintenance systemic corticosteroids (mOCS)] to prevent it from becoming uncontrolled or which remains uncontrolled despite this therapy (3). Patients with severe asthma experience a heavy burden of symptoms, exacerbations, and medication side effects leading to a decreased quality of life and considerable psychosocial impact $(1,4,5)$. Additionally, severe asthma has a significant economic impact on society. The total costs (direct, indirect, and intangible) are difficult to estimate, but the treatment costs alone are estimated to be $£ 2912-£ 4217$ ( $€ 3413-€ 4943)$ per person per year (6). Therefore, optimal treatment to control severe asthma is important.

Severe asthma is a heterogenic disease with several phenotypes, such as early onset atopic asthma, late onset eosinophilic asthma, neutrophilic asthma, and late onset obese asthma. Eosinophilic asthma accounts for $55 \%$ of severe asthma cases (7). Severe eosinophilic asthma is mostly late onset, characterized by frequent exacerbations and may only be controlled using maintenance systemic corticosteroids (mOCS). Persistent 
blood eosinophilia (an eosinophilic cell count of $>0.30 \times 10^{9} / \mathrm{L}$ when on high dose inhaled glucocorticoids or $>0.15 \times 10^{9} / \mathrm{L}$ when on mOCS) is a hallmark of this phenotype.

Mepolizumab is a humanized monoclonal antibody that binds to and inactivates interleukin-5 and effectively inhibits eosinophilic airway inflammation (8). Three randomized controlled trials (RCTs) showed clinical efficacy of mepolizumab $100 \mathrm{mg}$ subcutaneously as add-on treatment in patients with severe refractory eosinophilic asthma (9-11). Therefore, mepolizumab is approved and registered in The Netherlands as add-on medication for patients with severe refractory eosinophilic asthma since February 2016. As it is important to assess effectiveness and safety of novel treatments in real world practice, Dutch healthcare authorities and health insurance companies require healthcare professionals to collect data regarding mepolizumab efficacy and safety in clinical practice. This observational single center study was conducted to evaluate response rate, effectiveness, and safety in real world practice.

\section{Methods}

\section{Study design}

The MepOlizuMab Experience in the NeTherlands (MOMENT) study is a retrospective observational, longitudinal study in patients with severe eosinophilic asthma treated with mepolizumab in a tertiary referral center for severe asthma in Rotterdam, The Netherlands. Approval of the local Medical Ethics Committee was obtained.

From August 2018 until April 2019 patients 18 years or older, with severe eosinophilic asthma and who were treated with at least one dose of mepolizumab were randomly asked to participate in the study. All included patients provided written informed consent. Patients with COPD (Chronic Obstructive Pulmonary Disease) or EGPA (Eosinophilic Granulomatosis with PolyAngiitis) were excluded. Mepolizumab was administered every 4 weeks with a dose of $100 \mathrm{mg}$ subcutaneously.

Data were collected by the first two authors and randomly cross-checked, using Castor EDC (Castor Electronic Data Capture, Ciwit BV, Amsterdam, The Netherlands 2018).

Data were collected retrospectively 12 months before the initiation of mepolizumab, at the start of mepolizumab (baseline) and 2, 4, 6, 9, and 12 months from the initiation of mepolizumab. The personal data were anonymized and handled according to the General Data Protection Regulation.

\section{Objectives and statistics}

Demographic and other baseline characteristics were summarized using descriptive statistics.

\section{Primary objective}

The primary objective was to determine the response rate and (dis)continuation rate of mepolizumab, based on a global evaluation of the treatment effectiveness by the treating pulmonologist. Additionally, the reasons for discontinuation of mepolizumab were assessed.

\section{Secondary objectives}

A binominal logistic regression was performed to assess the effects of type of asthma (allergic eosinophilic versus non allergic eosinophilic asthma), eosinophil count, smoking behavior, and mOCS usage on the likelihood that mepolizumab was discontinued 12 months from baseline. These independent variables were used because these can theoretically influence the effect of mepolizumab: possibly not only eosinophilic inflammation, but also other pathways play an important role in the severity of allergic eosinophilic asthma resulting in a reduced effect of mepolizumab (12); an earlier study showed a close relationship between baseline blood eosinophil count and the effect of mepolizumab on exacerbation frequency (13); smoking reduces pulmonary eosinophil numbers and IL-5 concentrations which could reduce the effect of mepolizumab (14); patients with steroid resistant asthma may have a different immunophenotype compared to patients with steroid sensitive asthma (15).

The effects of mepolizumab on exacerbation rate, steroid-sparing effect, Asthma Control Questionnaire (ACQ-6) and lung function were analyzed in order to compare the effectiveness of mepolizumab in real world practice with the effectiveness reported in RCTs. The hypothesis is that the effectiveness in real world practice is similar to the effectiveness in RCTs.

The effect of mepolizumab on asthma exacerbations was evaluated by calculating the difference in the mean frequency of severe asthma exacerbations and hospitalizations due to asthma exacerbations prior and during treatment with mepolizumab. The null hypothesis that there was no difference in the mean frequencies prior and during treatment with mepolizumab was tested by paired samples $t$-test. Severe asthma exacerbations were defined as any severe peak of symptoms that required an increase in dose of mOCS for three days or more, a cycle of systemic corticosteroids, a visit to the emergency department, 
Table 1. Characteristics of the patients at baseline.

\begin{tabular}{|c|c|}
\hline \multicolumn{2}{|l|}{ Type of asthma-no. (\%) ${ }^{a}$} \\
\hline Allergic & $34(43.6)$ \\
\hline Non allergic & $44(56.4)$ \\
\hline \multicolumn{2}{|l|}{ Onset of asthma-no. (\%) } \\
\hline Early onset & $25(32.1)$ \\
\hline Late onset & $42(53.8)$ \\
\hline Unknown & $11(14.1)$ \\
\hline Mean age-years (range) & $54(20-83)$ \\
\hline Female gender-no. (\%) & $54(69.2)$ \\
\hline \multicolumn{2}{|l|}{ Ethnicity-no. (\%) } \\
\hline Caucasian & $67(85.9)$ \\
\hline Other/Mixed & $8(10.3)$ \\
\hline African origin & $3(3.8)$ \\
\hline BMI_median (range) & $28.4(20.9-57.7)$ \\
\hline Patients with obesity-no. $(\%)^{b}$ & $31(39.7)$ \\
\hline \multicolumn{2}{|l|}{ Smoking-no. (\%) } \\
\hline Never & $51(65.4)$ \\
\hline Former & $25(32.1)$ \\
\hline Smoker & $2(2.6)$ \\
\hline Glucocorticoid use-no. (\%) & $40(51.3)$ \\
\hline Daily glucocorticoid dose-median mg prednisolone equivalent (range) ${ }^{c}$ & $10(3-50)$ \\
\hline History of treatment with omalizumab-no. (\%) & $18(23.1)$ \\
\hline Severe exacerbations in previous year-mean no./patient (range) & $4.6(0-12)$ \\
\hline Hospitalization due to an exacerbation in previous year-no. (\%) & $26(33.3)$ \\
\hline Intubation due to an exacerbation in previous year-no. (\%) & $2(2.6)$ \\
\hline ACQ score-mean (SD) & $2.56( \pm 0.96)$ \\
\hline FEV1 post bronchodilation-mean percent of predicted value (SD) ${ }^{d}$ & $75.9( \pm 17.3)$ \\
\hline FEV1:FVC ratio post bronchodilation-mean percent (SD) & $66.3( \pm 11.9)$ \\
\hline FeNO—mean ppb (SD) & $53.3( \pm 39.8)$ \\
\hline Blood eosinophil count-median $10^{9} / \mathrm{L}(\mathrm{IQR})^{\mathrm{e}}$ & $0.40(0.20-0.67)$ \\
\hline \multicolumn{2}{|c|}{$\begin{array}{l}\text { The diagnosis allergic asthma was based on a history of symptoms that occur upon exposure to a particular aeroallergen, or persist- } \\
\text { ent symptoms and suspicion of exposure to a relevant allergen in the (home) environment, and the presence of specific lgE anti- } \\
\text { bodies to the inhalant allergen. } \\
\text { b Obesity is defined as a Body Mass Index (BMI) of } 30 \mathrm{~kg} / \mathrm{m}^{2} \text { or more. }\end{array}$} \\
\hline
\end{tabular}

hospitalization, mechanical ventilation, or intensive care unit admission.

The steroid-sparing effect of mepolizumab was evaluated by comparing the percentage of patients being treated with mOCS before, 6 months from and 12 months from the initiation of mepolizumab. The null hypothesis that there was no difference in the percentage of patients treated with mOCS at baseline compared to 6 and 12 months from the initiation of mepolizumab was tested by McNemar's test.

To evaluate the effect of mepolizumab on ACQ and lung function the mean ACQ, mean percent of predicted FEV1 post bronchodilation and mean FeNO before, 6 months from and 12 months from the initiation of mepolizumab were compared. The null hypothesis that there was no difference in the mean ACQ, mean percent of predicted FEV1 and mean FeNO at baseline compared to 6 and 12 months from the initiation of mepolizumab was tested by paired samples t-test. An improvement in ACQ of 0.5 or more was considered clinically relevant.

As there were some missing values in the data of ACQ and lung function (in particular FeNO), only patients without missing data were included when calculating a difference between two time points and when testing the null hypothesis.

Data of all adverse events were collected and reported in this study in order to comply with European pharmacovigilance regulations. Adverse events were also reported to Lareb, the national authority collecting and analyzing data regarding adverse events.

\section{Results}

\section{Patients}

A total of 78 patients with severe eosinophilic asthma were included in our study. The mean age was 54 years, 54\% was female, most patients had late onset non allergic asthma, the mean rate of severe exacerbations per year was 4.6 , and $51.3 \%$ of patients used mOCS. Table 1 shows the characteristics of the patients at baseline. The most common comorbidities were rhinosinusitis and nasal polyposis. Table 2 shows the comorbidities of the included patients at baseline. 
Table 2. Comorbidities of the patients at baseline.

\begin{tabular}{lc}
\hline Comorbidity no. (\%) & $38(48.7)$ \\
Rhinosinusitis & $24(30.8)$ \\
Nasal polyposis & $12(15.4)$ \\
Obstructive sleep apnea syndrome & $9(11.5)$ \\
Diabetes mellitus & $7(9.0)$ \\
Cardiovascular disease & $7(9.0)$ \\
Bronchiectasis & $7(9.0)$ \\
Depressive disorder & $6(7.7)$ \\
Frequent exacerbations requiring antibiotics & $6(7.7)$ \\
Gastroesophageal reflux disease & $4(5.1)$ \\
Malignancy & $5(6.4)$ \\
Complications due to glucocorticoid therapy-no. (\%) & \\
\hline a & pwo patients had localized breast cancer more than 5years ago. One \\
patient had localized bladder cancer (transitional cell carcinoma) more \\
than 5 years ago. One patient had an inverted papilloma of the nose. \\
${ }^{b}$ Diseases that were considered most likely due to glucocorticoid therapy: \\
one patient had a psychosis, one patient adrenal insufficiency, one \\
patient cataract, one patient liver function disorders due to a metabolic \\
syndrome and one patient an avascular necrosis.
\end{tabular}

\section{Response rate}

Mepolizumab therapy was considered successful and was therefore continued in $66(84.6 \%)$ and $59(75.6 \%)$ patients 6 and 12 months from the start of treatment, respectively. The reason for discontinuing mepolizumab was insufficient response in 14 (17.9\%) patients. In $4(5.1 \%)$ patients the combination of insufficient response and adverse events (an increase in allergic symptoms in two patients, fever in one patient, low back pain and malaise in 1 patient) was the reason for ceasing mepolizumab therapy. One (1.3\%) patient had abdominal pain due to an Entamoeba histolytica and Helicobacter pylori infection 6 months from the start of mepolizumab therapy upon which mepolizumab was stopped temporarily. After successful treatment of the infection the patient preferred continuing mOCS over mepolizumab, which led to definitive discontinuation of mepolizumab in this patient.

Of the 19 patients that discontinued mepolizumab, 17 (89.5\%) proceeded treatment with another biological agent (10 patients reslizumab, 4 benralizumab, 2 dupilumab, 1 omalizumab) after discontinuing mepolizumab treatment.

\section{Risk factors for discontinuation}

Patients with nonallergic asthma were 3.3 times (CI $1.1-10.4 ; p=0.042$ ) less likely to have stopped mepolizumab therapy 12 months from baseline compared to patients with allergic asthma. The other variables did not show a statistically significant effect on this outcome.

\section{Asthma exacerbations}

The mean rate of severe asthma exacerbations was 4.6 (range 0-12) during the year prior to the start of mepolizumab. During mepolizumab therapy, this rate was 1.4 per patient year (range $0-6$ ). This is a decrease of 3.2 (CI 2.5-4.1; $p<0.001$ ) severe exacerbations per patient year compared to the number of severe exacerbations in the year prior to mepolizumab treatment. The mean number of hospital admissions due to an asthma exacerbation was 0.35 per patient year (range 0-5) during treatment with mepolizumab compared to 0.77 (range 0-10) during the year prior to mepolizumab treatment, this is a mean difference of 0.42 (CI $0.17-0.68 ; p=0.001$ ). There were no patients requiring intubation due to an asthma exacerbation during mepolizumab treatment.

\section{Systemic glucocorticoid maintenance therapy}

At baseline, 40 (51.3\%) patients were treated with mOCS. At 6 months from baseline 24.3\% (compared to baseline $p<0.001$ ) of the patients still being treated with mepolizumab were on mOCS. This percentage was $15.4 \%$ (compared to baseline $p<0.001$ ) 12 months from baseline.

\section{ACQ}

The mean ACQ at baseline was 2.56 (SD \pm 0.96 ). At 6 months from baseline the ACQ of the patients still using mepolizumab decreased 0.84 points (CI 0.61 $1.07 ; p<0.001)$. At 12 months from baseline, the ACQ was 0.80 points lower compared to baseline (CI $0.49-1.12 ; p<0.001$ ).

\section{Lung function}

The mean percent of predicted FEV1 post bronchodilation (SD) was $75.9( \pm 17.3)$ at baseline. The mean absolute increase of the percentage of predicted FEV1 was 2.7 (CI -0.6 to $5.9 ; p=0.109$ ) 6 months from baseline. At 12 months from baseline, this change was 3.7 (CI $0.3-7.2 ; p=0.034$ )\% of predictive value.

The mean FeNO (SD) was $53.3( \pm 39.8) \mathrm{ppb}$ at baseline. The FeNO decreased $8.5 \mathrm{ppb}$ (CI -22.6 to $5.6 ; p=0.231)$ and $6.7 \mathrm{ppb}(\mathrm{CI}-20$ to $6.7 ; p=0.318)$ 6 and 12 months from baseline, respectively.

\section{Adverse events}

Adverse events were reported by 37 patients (47.4\%). Adverse events that occurred in at least $2(2.6 \%)$ patients are listed in Table 3. Data of all adverse events are provided in Appendix Table A1. Headache was the most frequently reported adverse event. In 
Table 3. Adverse events. ${ }^{a}$

\begin{tabular}{lc}
\hline Adverse event & Number of patients (\%) \\
\hline Headache & $7(9.0)$ \\
Low back pain & $6(7.7)$ \\
Urinary tract infection & $5(6.4)$ \\
Spinal disc herniation & $3(3.9)$ \\
Nausea & $3(3.9)$ \\
Fatigue & $3(3.9)$ \\
Vertigo & $2(2.6)$ \\
Increase of allergic symptoms & $2(2.6)$ \\
Insomnia & $2(2.6)$ \\
\hline aAdverse events that occurred in at least two patients are listed in \\
this table.
\end{tabular}

most patients, the onset of headache was shortly after the first dose of mepolizumab. The headache resolved in all patients during the first 6 months from baseline. No serious adverse events considered to be related to mepolizumab were reported during our study.

\section{Discussion}

Several RCTs demonstrated a beneficial effect of mepolizumab add-on therapy in patients with severe eosinophilic asthma (9-11). However, little is known about the response rate, efficacy, and safety of mepolizumab in daily practice. To our knowledge, our study is the largest study to date to assess response rate, efficacy as well as safety in patients with severe eosinophilic asthma who are treated with at least one dose of mepolizumab.

In our study $75.6 \%$ of patients continued mepolizumab therapy 1 year from the initiation of mepolizumab. The main reason for discontinuation of mepolizumab was insufficient response based on global evaluation of treatment effect. Other reasons were the combination of insufficient response and adverse events, and patient's decision. The mean drop-out rate in RCTs was $9 \%$ with a mean treatment duration of 8.5 months (16). Reasons for drop-out in RCTs were "other" (not specified; $36.5 \%$ of the patients that dropped out), adverse events (23.7\%), lack of efficacy (21.3\%), and personal decision (19.5\%) (16). To our knowledge, three real world studies assessed drop-out rate (studies only including responders (patients treated with mepolizumab for at least 12 months) were excluded): Zhang et al. (17) reported a drop-out rate of $20 \%$ in 30 patients with a mean treatment duration of 5.4 months. Drop-out was due to insufficient response (50\%) and adverse events (50\%). Lombardi et al. (16) reported a drop-out rate of $4 \%$ in 143 patients during the first year of mepolizumab treatment, mainly due to insufficient response (83\%). Ntakoula et al. (18) reported a drop-out rate of $36 \%$ in 11 patients with a follow up of 6 months. Drop-out was due to lack of response (50\%), myalgia (25\%), and personal reasons (25\%). As RCTs are conducted under rigorously controlled conditions, the drop-out rate is expected to be higher in daily practice. Therefore, a drop-out rate of approximately 25\% 1 year from the start of mepolizumab seems reasonable. In particular, since alternative biological treatments became available. Benralizumab, which causes a nearly complete elimination of airway eosinophils (19), and reslizumab, which could have better systemic uptake in some patients (as it is administered intravenous), could theoretically be beneficial in some patients with insufficient response to mepolizumab. However, research regarding this topic is lacking. The drop-out rate described in our study is in line with omalizumab data, which show a drop-out rate of $7.1-19.4 \%$ and $32 \%$ in RCTs and the real world NEONet database, respectively (20).

Bel et al. (10) described that after 20 weeks of treatment $14 \%$ of their patients had a $100 \%$ reduction in mOCS use. In our cohort $27 \%$ of patients could stop the mOCS 6 months after the initiation of mepolizumab, increasing up to $35.9 \%$ after 12 months. The annualized rates of exacerbations were significantly reduced in the mepolizumab group, with our study showing a reduction of $70 \%$, which is higher compared to the RCTs (32, 53, and 58\% reduction) (9-11). Patients in our study appear to have more severe asthma compared to the patients included in the RCTs, as our patients had more frequent asthma exacerbations and used more often mOCS during the year prior to initiation of mepolizumab treatment. This could be due to patient selection, as patients with a significant smoking history or considerable comorbidity were excluded in RCTs. Patients with more severe asthma might benefit even more from mepolizumab therapy, which possibly explains the greater improvement in asthma exacerbation rate and mOCS usage in our study compared to RCTs.

We found a statistically significant and clinically relevant improvement of ACQ 6 and 12 months from baseline. Three RCTs assessed ACQ. All reported a statistically significant improvement of ACQ, but this was clinically relevant in only one trial (9-11). Lung function improved statistically significant but clinically marginal during mepolizumab therapy. FeNO remained high in many patients that responded to mepolizumab therapy.

Interestingly, $9 \%$ of patients discontinued mepolizumab later than 6 months from the initiation of mepolizumab. As the improvements of ACQ and lung function are stable, and the percentage of patients 
using mOCS is even declining when comparing the data 6 and 12 months from the initiation of mepolizumab, a general decrease of the efficacy of mepolizumab over time is not likely. The decline in the percentage of patients using mOCS over time is not due to cessation of mepolizumab by mOCS users, as the percentage of patients who used mOCS is smaller in the group of patients that discontinued mepolizumab compared to patients that continued mepolizumab. Neutralizing anti-drug antibodies are probably rare (21). The decrease in response rate 6 months from the initiation of mepolizumab could be due to patients who show a partial response that is not convincing at first, and in which the period of evaluation is therefore extended. To better understand the long term effectiveness of mepolizumab, a study with a follow-up of multiple years is required.

No serious adverse events considered to be related to mepolizumab were reported during our study, which endorses mepolizumab's favorable safety profile (22). The most frequently reported adverse events were headache, low back pain, and urinary tract infection, which is consistent with earlier RCTs.

In our study, patients with nonallergic eosinophilic asthma were less likely to discontinue mepolizumab compared to patients with allergic eosinophilic asthma. This finding could suggest that not only ILC2-driven eosinophilic inflammation, but also other factors [possibly specific IgE or other $\mathrm{T}_{\mathrm{H}} 2$-cytokines (12)] play an important role in the pathophysiology of allergic eosinophilic asthma. However, this finding should be interpreted with caution as this was tested with a logistic regression analysis in a retrospective study without correction for multiple testing, response rate is a subjective endpoint (based on a global evaluation of treatment effect by the treating pulmonologist) and, to our knowledge, no other studies tested this hypothesis. Further research to assess this hypothesis is necessary.

As this study is a retrospective cohort study, data were not collected prospectively on standardized time points, and patients acted as their own control, increasing the risk of confounding: asthma is a dynamic disease, which could result in a spontaneous improvement of symptoms. And as our hospital is a tertiary referral center for severe asthma, some included patients where referred to our hospital for mepolizumab therapy. Although conventional treatment was optimized before starting mepolizumab, the effects of other treatments could magnify the results reported in this study. Also, there were some missing values in the data of ACQ and lung function. It is, however, important to assess the effectiveness of a new treatment in real world practice, as study populations in RCTs only represent a small proportion of the regular severe asthma population (23). Real world cohort studies, therefore, contribute valuable complementary information to the current knowledge.

How long treatment with mepolizumab should be continued is unknown. There are also insufficient data to date concerning long term side effects. More research addressing this topic is therefore needed.

In conclusion, three out of four patients were still on mepolizumab add-on therapy one year after starting mepolizumab for severe eosinophilic asthma. Our study confirms that mepolizumab decreases the rate of severe asthma exacerbations and hospital admissions due to asthma exacerbations, improves asthma control, reduces mOCS usage and has a favorable safety profile in real world practice.

\section{Acknowledgments}

GlaxoSmithKline was involved in designing this study. GlaxoSmithKline was not involved in patient inclusion, data collection, data analysis, interpretation of the results, the process of writing this paper or the decision to submit this article for publication.

\section{Data availability statement}

The data that support the findings of this study are available on request from the corresponding author, GJ Braunstahl. The data are not publicly available to ensure the privacy of the research participants.

\section{Declaration of interest}

JJ van Toor declares no conflicts of interest. SC van der Mark declares no conflicts of interest. JH Kappen has received grant/research support for consultations and/or speaking at conferences from Novartis, ALK-Abello, GlaxoSmithKline, Chiesi, AstraZeneca, Boerhinger, Sanofi, Mylan. JCCM in 't Veen has received grant/research support for consultations and/or speaking at conferences from Novartis, Boehringer Ingelheim, GlaxoSmithKline, Chiesi, AstraZeneca, and Sanofi. G-J Braunstahl has received grant/ research support for consultations and/or speaking at conferences from Novartis, ALK-Abello, Meda Pharma, GlaxoSmithKline, Takeda, Chiesi, Teva, AstraZeneca, and MSD.

\section{Funding}

This study is sponsored by a grant [grant number ISS209477] of GlaxoSmithKline. 


\section{ORCID}

Jermo Johannes van Toor (D) http://orcid.org/0000-00017315-3436

\section{References}

1. Global Initiative for Asthma. Global strategy for asthma management and prevention. Available from: https://ginasthma.org/gina-reports/ [last accessed September 2019].

2. Hekking PP, Wener RR, Amelink M, Zwinderman $\mathrm{AH}$, Bouvy ML, Bel EH. The prevalence of severe refractory asthma. J Allergy Clin Immunol. 2015; 135(4):896-902. doi:10.1016/j.jaci.2014.08.042.

3. Chung KF, Wenzel SE, Brozek JL, Bush A, Castro M, Sterk PJ, Adcock IM, Bateman ED, Bel EH, Bleecker ER, et al. International ERS/ATS guidelines on definition, evaluation and treatment of severe asthma. Eur Respir J. 2014;43(2):343-373. doi:10.1183/09031936.00202013.

4. Foster JM, McDonald VM, Guo M, Reddel HK. I have lost in every facet of my life": the hidden burden of severe asthma. Eur Respir J. 2017;50(3):1700765. doi:10.1183/13993003.00765-2017.

5. Hossny E, Caraballo L, Casale T, El-Gamal Y, Rosenwasser L. Severe asthma and quality of life. World Allergy Organ J. 2017;10:28. doi:10.1186/s40413017-0159-y.

6. O’Neill S, Sweeney J, Patterson CC, Menzies-Gow A, Niven R, Mansur AH, Bucknall C, Chaudhuri R, Thomson NC, Brightling CE. The cost of treating severe refractory asthma in the UK: an economic analysis from the British Thoracic Society Difficult Asthma Registry. Thorax. 2015;70:376-378. doi:10. 1136/thoraxjnl-2013-204114.

7. Schleich F, Brusselle G, Louis R, Vandenplas O, Michils A, Pilette C, Peche R, Manise M, Joos G. Heterogeneity of phenotypes in severe asthmatics. The Belgian Severe Asthma Registry (BSAR). Respir Med. 2014;108(12):1723-1732. doi:10.1016/j.rmed.2014.10.007.

8. Flood-Page P, Swenson C, Faiferman I, Matthews J, Williams M, Brannick L, Robinson D, Wenzel S, Busse W, Hansel TT, on behalf of the International Mepolizumab Study Group, et al. A study to evaluate safety and efficacy of mepolizumab in patients with moderate persistent asthma. Am J Respir Crit Care Med. 2007; 176(11):1062-1071. doi:10.1164/rccm.200701-085OC.

9. Pavord ID, Korn S, Howarth P, Bleecker ER, Buhl R, Keene ON, Ortega H, Chanez P. Mepolizumab for severe eosinophilic asthma (DREAM): a multicenter, double-blind, placebo-controlled trial. Lancet. 2012; 380(9842):651-659. doi:10.1016/S0140-6736(12)60988-X.

10. Bel EH, Wenzel SE, Thompson PJ, Prazma CM, Keene ON, Yancey SW, Ortega HG, Pavord ID. Oral glucocorticoid-sparing effect of mepolizumab in eosinophilic asthma. N Engl J Med. 2014;371(13): 1189-1197. doi:10.1056/NEJMoa1403291.

11. Ortega HG, Liu MC, Pavord ID, Brusselle GG, FitzGerald JM, Chetta A, Humbert M, Katz LE, Keene $\mathrm{ON}$, Yancey SW, et al. Mepolizumab treatment in patients with severe eosinophilic asthma. N Engl J Med. 2014;371(13):1198-1207. doi:10.1056/NEJMoa1403290.
12. Kuruvilla ME, Eun-Hyung Lee F, Lee GB. Understanding asthma phenotypes, endotypes, and mechanisms of disease. Clinic Rev Allerg Immunol. 2019;56(2):219-233.

13. Ortega HG, Yancey SW, Mayer B, Gunsoy NB, Keene ON, Bleecker ER, Brightling CE, Pavord ID. Severe eosinophilic asthma treated with mepolizumab stratified by baseline eosinophil thresholds: a secondary analysis of the DREAM and MENSA studies. Lancet Respir Med. 2016;4(7):549-556. doi:10.1016/S22132600(16)30031-5.

14. Tilp C, Bucher H, Haas H, Duechs MJ, Wex E, Erb KJ. Effects of conventional tobacco smoke and nicotine-free cigarette smoke on airway inflammation, airway remodelling and lung function in a triple allergen model of severe asthma. Clin Exp Allergy. 2016;46(7): 957-972.

15. Chambers ES, Nanzer AM, Pfeffer PE, Richards DF, Timms PM, Martineau AR, Griffiths CJ, Corrigan CJ, Hawrylowicz CM. Distinct endotypes of steroid-resistant asthma characterized by IL-17A ${ }^{\text {high }}$ and IFN-y ${ }^{\text {high }}$ immunophenotypes: potential benefits of calcitriol. J Allergy Clin Immunol. 2015;136(3):628-637.e4. doi:10. 1016/j.jaci.2015.01.026.

16. Lombardi C, Bagnasco D, Caruso C, D’Amato M, Menzella F, Milanese M, Senna G, Canonica GW, Passalacqua G. Analysis of the drop-out rate in patients receiving mepolizumab for severe asthma in real life. Pulm Pharmacol Ther. 2019;54:87-89. doi:10. 1016/j.pupt.2018.12.003.

17. Zhang P, Vitari CA, Wenzel SE. Real world effectiveness and safety of anti-IL-5/mepolizumab in an academic severe asthma clinic. Am J Respir Care Med. 2017;195:A3187.

18. Ntakoula M, Fokoloros C, Pasali M, Aggelides X, Makris M. Real life data on treatment response to mepolizumab in patients with severe eosinophilic asthma. Allergy. 2019;74:376-853.

19. Laviolette $M$, Gossage DL, Gauvreau G, Leigh R, Olivenstein R, Katial R, Busse WW, Wenzel S, Wu Y, Datta V, et al. Effects of benralizumab on airway eosinophils in asthmatic patients with sputum eosinophilia. J Allergy Clin Immunol. 2013;132(5): 1086-1096. doi:10.1016/j.jaci.2013.05.020.

20. Caminati M, Senna G, Stefanizzi G, Bellamoli R, Longhi S, Chieco-Bianchi F, Guarnieri G, Tognella S, Olivieri M, Micheletto C, on behalf of North East Omalizumab Network study group, et al. Drop-out rate among patients treated with omalizumab for severe asthma: literature review and real-life experience. BMC Pulm Med. 2016;16(1):128. doi:10.1186/ s12890-016-0290-5.

21. Ortega H, Meyer E, Brusselle G, Asano K, Price R, Prazma C, Albers F, Yancey S, Gleich G. Immunogenicity of mepolizumab in patients with severe eosinophilic asthma: experience from the clinical development program. Eur Respir J. 2018;52:OA1650. doi:10.1183/13993003.congress-2018.OA1650.

22. Lugogo N, Domingo C, Chanez P, Leigh R, Gilson MJ, Price RG, Yancey SW, Ortega HG. Long-term efficacy and safety of mepolizumab in patients with severe eosinophilic asthma: a multi-center, open-label, 
phase IIIb study. Clin Ther. 2016;38(9):2058-2070. doi:10.1016/j.clinthera.2016.07.010.

23. Herland K, Akselsen JP, Skjønsberg OH, Bjermer L. How representative are clinical study patients with asthma or COPD for a larger "real life" population of patients with obstructive lung disease?. Respir Med. 2005;99(1):11-19. doi:10.1016/j.rmed.2004. 03.026 .

\section{Appendix}

Table A1. All reported adverse events.

\begin{tabular}{|c|c|}
\hline Adverse event & Number of patients (\%) \\
\hline Headache & $7(9.0)$ \\
\hline Low back pain & $6(7.7)$ \\
\hline Urinary tract infection & $5(6.4)$ \\
\hline Spinal disc herniation & $3(3.9)$ \\
\hline Nausea & $3(3.9)$ \\
\hline Fatigue & $3(3.9)$ \\
\hline Vertigo & $2(2.6)$ \\
\hline Increase of allergic symptoms & $2(2.6)$ \\
\hline Insomnia & $2(2.6)$ \\
\hline Hematoma & $1(1.3)$ \\
\hline Syncope of unknown origin & $1(1.3)$ \\
\hline Periorbital puffiness & $1(1.3)$ \\
\hline Xerostomia & $1(1.3)$ \\
\hline Lateral epicondylitis & $1(1.3)$ \\
\hline Epistaxis & $1(1.3)$ \\
\hline Flu like symptoms during the first 2 days following mepolizumab injection & $1(1.3)$ \\
\hline Helicobacter pylori and Entamoeba histolytica infection & $1(1.3)$ \\
\hline Polymorphous light eruption & $1(1.3)$ \\
\hline Hypertension & $1(1.3)$ \\
\hline Increase of asthma symptoms during the first couple of days following mepolizumab injection & $1(1.3)$ \\
\hline Pruritus & $1(1.3)$ \\
\hline Knee pain & $1(1.3)$ \\
\hline Fever & $1(1.3)$ \\
\hline Chronic low grade noninvasive fungal rhinosinusitis (aspergillus fumigatus) & $1(1.3)$ \\
\hline Melanoma & $1(1.3)$ \\
\hline Meniscus injury & $1(1.3)$ \\
\hline Nummular dermatitis & $1(1.3)$ \\
\hline Obstipation & $1(1.3)$ \\
\hline Paroxysmal atrial fibrillation & $1(1.3)$ \\
\hline Palpitations & $1(1.3)$ \\
\hline Pharyngitis & $1(1.3)$ \\
\hline Pain in hands and legs during the first couple of days following mepolizumab injection & $1(1.3)$ \\
\hline Angina pectoris & $1(1.3)$ \\
\hline Restless legs syndrome and periodic limb movement disorder & $1(1.3)$ \\
\hline Thrombophlebitis & $1(1.3)$ \\
\hline Urolithiasis & $1(1.3)$ \\
\hline Forgetfulness & $1(1.3)$ \\
\hline
\end{tabular}

